\title{
Methylation regulates Tau aggregation and neuronal health in Alzheimer's disease
}

\author{
Abhishek Ankur Balmik \\ Subashchandrabose Chinnathambi
}

\section{Video Byte}

Keywords: Tau, methylation, methyltransferases, post-translational modifications, epigenetics, aggregation, Alzheimer's disease, neurodegeneration, brain, Cell Communication and Signaling

Posted Date: October 14th, 2021

DOl: https://doi.org/10.21203/rs.3.rs-967805/v1

License: (c) (1) This work is licensed under a Creative Commons Attribution 4.0 International License. Read Full License 


\section{Abstract}

Alzheimer's disease (AD) is associated with the misfolding of two major proteins, causing the accumulation of toxic protein aggregates. Amyloid- $\beta$ aggregates extracellularly, forming plaques, and Tau aggregates intracellularly, forming neurofibrillary tangles. Post-translational modifications (PTMs) are important for the regulation of Tau's function, but an imbalance in PTMs may lead to abnormal Tau function and aggregation. While methylation is an important PTM for Tau in its physiological state, the lysine methylation signature changes in Tau's pathological form. These alterations may affect the intramolecular forces within the Tau molecule, resulting in altered conformations, and methylation can also interact with other PTMs, affecting Tau function and stability. Although more investigation is needed, it appears that in addition to post-translational methylation, DNA methylation also affects Tau regulation. In addition to its specific effects on Tau, DNA methylation also globally affects epigenetic regulation and metabolism in neurodegenerative diseases. Methylation is important in AD as both an epigenetic regulatory marker and a PTM, although the precise mechanisms of both remain unclear. Better understanding the varied roles of methylation in Tau aggregation and overall neuronal health will help researchers to develop therapies to better prevent and treat AD. 